\title{
Zur Rechtsfigur des de facto-Organs im Recht der Staatenverantwortlichkeit
}

\author{
Astrid Epiney
}

\begin{abstract}
Dieser Beitrag wurde erstmals wie folgt veröffentlicht:
Astrid Epiney, Zur Rechtsfigur des de facto-Organs im Recht der Staatenverantwortlichkeit, FS Michael Bothe, Baden-Baden/Sankt Gallen 2008, S. 883896. Es ist möglich, dass die Druckversion - die allein zitierfähig ist - im Verhältnis zu diesem Manuskript geringfügige Modifikationen enthält.
\end{abstract}

\section{Problemstellung}

Die Rechtsfigur des sog. de facto-Organs ist seit den Anfängen der Entwicklung des Rechts der Staatenverantwortlichkeit ein immer wieder praktisch relevantes ${ }^{1}$ und in der Lehre erörtertes ${ }^{2}$ Thema. Dabei geht die Grundidee der Entwicklung des de facto-Organs bzw. der Zurechnung seines Verhaltens zum Staat dahin, dass den Staaten nicht nur solche Handlungen zugerechnet ${ }^{3}$ werden sollen, die von „offiziellen“ Staatsorganen ausgehen, sondern auch solche von an sich als Private ${ }^{4}$ einzuordnenden Personen, die in der einen oder anderen Form faktisch „für“ den betreffenden Staat agieren. Obwohl über diesen Grundgedanken Einigkeit besteht, stößt es nach wie vor auf Schwierigkeiten, die genauen Voraussetzungen der Zurechnung des jeweiligen Verhaltens zum Staat im Falle „faktisch für den Staat agierender

$1 \quad$ Vgl. etwa aus der Judikatur bereits ICJ Rep. 1980, 3, Ziff. 58 ff. (Teheraner Geiselfall); ICJ Rep. 1986, 14, Ziff. 86 ff. (Military and Paramilitary Activities in and against Nicaragua); ICTY, IT-94-1, Trial Chamber, Opinion and Judgement, 7.5.1997, Ziff. 117 ff., 588 ff. (Tadic); IT-94-1-A, ICTY, Appeals Chamber, Judgement, 15.7.1999 (Tadic); ICTY, Appeals Chamber, Judgement, 20.2.2001 (Celebici); RIAA VI, 160 (Zafiro); RIAA IV, 265 (Stephens); RIAA VIII, 84 (Black Tom and Kingsland); 17 IranUSCTR, 1987-IV, 92 (Yeager); s. darüber hinaus den Überblick über die völkerrechtliche Praxis bei Jörn Griebel, Die Zurechnungskategorie der de facto-Organe im Recht der Staatenverantwortlichkeit, 2004, $128 \mathrm{ff}$.

2 Vgl. z.B. Griebel, de facto-Organe (Fn. 1); Claus Kress, L'organe de facto en droit international public réflexions sur l'imputation à l'Etat de l'acte d'un particulier à la lumière des développements récents, RGDIP 2001, 93 ff.; André J. de Hoogh, Articles 4 and 8 of the 2001 ILC Articles on State Responsibility, the Tadic Case and Attribution of Acts of Bosnian Serb Authorities to the Federal Republic of Yugoslavia, BYIL 2001, 255 ff.; Katja S. Ziegler, Fluchtverursachung als völkerrechtliches Delikt, 2002, 116 ff.; Luigi Condorelli, L'imputation à l'Etat d'un fait internationalement illicite: solutions classiques et nouvelles tendances, RdC 1984 VI, 96 ff.: Gregory Townsend, State Responsibility for Acts of De Facto Agents, Arizona Journal of International and Comparative Law 1997, $635 \mathrm{ff}$.

3 Zur Zurechnung als Element des Völkerrechtsdelikts Art. 2 ILC-Entwurf zur Verantwortlichkeit der Staaten für völkerrechtswidrige Handlungen, verabschiedet von der ILC im Jahr 2001, vgl. UN Doc. A/Res./56/83 (im Folgenden ILC-Entwurf). Aus der Literatur zu den Elementen des Völkerrechtsdelikts m.w.N. nur Ziegler, Fluchtverursachung (Fn. 2), 85 ff.; Andreas Felder, Die Beihilfe im Recht der völkerrechtlichen Staatenverantwortlichkeit, 2007, 46 ff.

4 Deren Verhalten den Staaten grundsätzlich nicht zugerechnet werden kann. Vgl. ausführlich hierzu bereits Astrid Epiney, Die völkerrechtliche Verantwortlichkeit von Staaten für rechtswidriges Verhalten im Zusammenhang mit Aktionen Privater, 1992, passim; Joachim Wolf, Die Haftung der Staaten für Privatpersonen nach Völkerrecht, 1997, passim. 
Personen“ zu umschreiben und zu präzisieren. So fragt es sich etwa, welche Intensität die staatliche Kontrolle haben muss, ob und inwieweit ultra vires-Handlungen auch bei de factoOrganen zugerechnet werden können oder in welchem Verhältnis der in Art. 8 ILC-Entwurf geregelte Zurechnungsgrund $\mathrm{zu}$ anderen Zurechnungsgründen, wie insbesondere dem in Art. 11 ILC-Entwurf geregelten Zurechnungsgrund der „Übernahme“ privaten Verhaltens als eigenes Verhalten (der letztlich auch als eine Kategorie von de facto-Organen angesehen werden kann), steht.

Vor diesem Hintergrund soll im Folgenden eine „Auslegeordnung“ für die Zurechnung des Verhaltens von de facto-Organen zum Staat entwickelt und auf diese Weise die genaue Tragweite dieses Zurechnungsgrundes präzisiert werden. Dabei kann zwischen den Voraussetzungen für das Eingreifen des Zurechnungsgrundes des Handelns eines de factoOrgans im Sinne des Art. 8 ILC-Entwurf (II.) und der Abgrenzung zu anderen Zurechnungsgründen (III.) unterschieden werden, wobei hier insbesondere Art. 11 ILCEntwurf von Bedeutung ist. Der Beitrag endet mit einigen kurzen Schlussbemerkungen zur Rolle der Rechtsfigur des de facto-Organs im Recht der Staatenverantwortlichkeit (IV.).

Zwar ist in Bezug auf einige hier zentrale Fragen die völkerrechtliche Praxis nicht immer klar. Dies ändert jedoch nichts daran, dass völkerrechtliche Regeln formuliert werden können, denn diese können sich auch aus von Vernunft und Logik geleiteten dogmatischen Überlegungen, die an einem konkreten Rechtsphänomen orientiert und damit seiner Problematik angemessen sind, ergeben ${ }^{5}$.

\section{Zu den tatbestandlichen Voraussetzungen der Zurechnung des Verhaltens eines de facto-Organs im Sinne des Art. 8 ILC-Entwurf}

In der völkerrechtlichen Praxis ist es - wie bereits eingangs erwähnt - anerkannt, dass das Handeln Privater einem Staat dann zuzurechnen ist, wenn Private im Auftrag des Staates agieren oder letzterer die Kontrolle über das jeweilige private Verhalten ausübt und die Privaten damit faktisch für den Staat tätig werden. Die ILC formulierte diesen Grundsatz in Art. 8 ILC-Entwurf, der Völkergewohnheitsrecht wiedergibt ${ }^{6}$, wobei die Bestimmung aber verschiedene auslegungsbedürftige Rechtsbegriffe enthält, so dass sie für sich allein denn auch nicht alle Fragen nach der genauen Tragweite dieses Zurechnungsgrundes zu beantworten vermag.

Art. 8 ILC-Entwurf

\footnotetext{
5 Vgl. hierzu Albert Bleckmann, Die Aufgaben einer Methodenlehre des Völkerrechts: Probleme der Rechtsquellenlehre im Völkerrecht, 1978, 40 ff.

6 Vgl. Kommentar der ILC, YbILC 1974 II 1, 283 ff:; s. auch schon Epiney, Völkerrechtliche Verantwortlichkeit (Fn. 4), 99 ff.; Ziegler, Fluchtverursachung (Fn. 2), 116 ff.; Griebel, de facto-Organe (Fn. 1), 47 ff.; Rüdiger Wolfrum, State Responsibility for Private Actors: an old Problem of Renewed Relevance, in: Essays in Memory of Oscar Schachter, 2005, 423 (427 ff.), jeweils mit zahlreichen Nachweisen aus der Praxis.
} 
„Das Verhalten einer Person oder Personengruppe ist als Handlung eines Staates im Sinne des Völkerrechts zu werten, wenn die Person oder Personengruppe dabei faktisch im Auftrag oder unter der Leitung oder Kontrolle dieses Staates handelt." ${ }^{\text {"7 }}$

Die Formulierung des Art. 8 ILC-Entwurfs lässt bereits die beiden Alternativen erkennen, bei denen eine Zurechnung eines Verhaltens nach Art. 8 ILC-Entwurf erfolgen kann: die Beauftragung einerseits (1.) und die tatsächliche staatliche Leitung oder Kontrolle andererseits (2.).

\section{Zur Beauftragung}

Zunächst ist eine Zurechnung immer dann anzunehmen, wenn ein „normales“ staatliches Organ Private beauftragt, bestimmte Handlungen vorzunehmen. Diese Alternative ist in der Staatenpraxis klar anerkannt ${ }^{8}$ und wurde schon von Berichterstatter Ago hervorgehoben'. Versucht man eine Präzisierung der Voraussetzungen dieses Zurechnungstatbestandes, so ist einerseits von der grundsätzlich notwendigen „Herrschaft“ des jeweiligen Verhaltens durch den beauftragenden Staat auszugehen; andererseits ist der systematische Zusammenhang dieser Alternative zur zweiten Alternative des Art. 8 ILC-Entwurfs zu beachten. Auf dieser Grundlage und in Anknüpfung an die völkerrechtliche Praxis ${ }^{10}$ können die Voraussetzungen bzw. Charakteristika dieses Zurechnungsgrundes wie folgt präzisiert werden:

- Der „Auftrag“ muss konkretisiert worden sein; andernfalls ist die für die Zurechnung notwendige Verbindung zwischen dem Verhalten der agierenden Person und dem Staat nicht hinreichend eng. Denn lediglich eine Art vorherige allgemeine Zustimmung zu einer bestimmten Art von Aktivitäten durch einen Staat vermag die für eine Zurechnung grundsätzlich privaten Verhaltens jedenfalls notwendige enge Beziehung zwischen dem Staat und dem Verhalten Privater nicht herzustellen. Daher reichen etwa allgemeine Aufforderungen, eine bestimmte Politik zu verfolgen, jedenfalls nicht für die Bejahung einer Zurechnung auf der Grundlage des Art. 8 ILC-Entwurfs aus. Fraglich könnte jedoch in diesem Zusammenhang sein, wie konkret die Beauftragung nun tatsächlich sein muss, sowohl was die betroffenen Personen als auch was die genaue Durchführung der Mission betrifft. Insbesondere der Vergleich mit der 2. Alternative des Art. 8 ILCEntwurf, in dem unstreitig eine gewisse Kontrolle verlangt wird, spricht dafür, dass ein Beauftragung im Sinne des Art. 8 ILC-Entwurfs von Vornherein nur dann vorliegen

\footnotetext{
7 "The conduct of a person or group of persons shall be considered an act of a State under international law if the person or group of persons is in fact acting on the instructions of, or under the direction or control of, that State in carrying out the conduct."

8 Insbesondere der IGH fragte im Teheraner Geiselfall für die erste Phase des Geschehens nach der Beauftragung der Akteure (die abgelehnt wurde), vgl. ICJ Rep. 1980, 3, Ziff. 57 ff.; vgl. ansonsten auch IT-94-1-A, ICTY, Appeals Chamber, Judgement, 15.7.1999 (Tadic); RIAA VIII, 84 (Black Tom and Kingsland).

$9 \quad$ Vgl. YbILC 1971 II 1, 263 ff. (Dritter Bericht zur Staatenverantwortlichkeit).

10 Vgl. insbesondere ICJ Rep. 1986, 3, Ziff. 57 ff. (Nicaragua); 16 Iran-USCTR, 76 ff. (Short).
} 
kann, wenn der Beauftragte identifiziert und individualisert ist und selbst einen persönlichen Auftrag erhalten hat, der sich seinerseits auf eine konkrete Tat bzw. ein konkretes Verhalten - und nicht eine allgemeine Richtung der Tätigkeit bezieht ${ }^{11}$. Die zuletzt genannte Voraussetzung dürfte allerdings immer schon dann gegeben sein, wenn das Ziel der Beauftragung klar formuliert ist; eine Präzisierung der einzusetzenden Mittel hingegen dürfte nicht notwendig sein; der Staat muss m.a.W. als Urheber des in Frage stehenden Verhaltens erscheinen.

Diese Voraussetzungen können an folgenden Fallkonstellationen bzw. Fallgruppen illustriert werden: Allgemeine Aufrufe des Staates X, den Schriftsteller S zu töten, vermögen keine Zurechnung eines möglichen Tötungsdelikts durch den Attentäter A zu begründen ${ }^{12}$. Wenn der Staat X den Attentäter A beauftragt, den Schriftsteller S zu töten, ohne zu präzisieren, wie dies geschehen soll, ist hingegen von einer Zurechnung auszugehen. Fraglich könnte sein, ob es für die Bejahung einer hinreichend präzisen Beauftragung ausreicht, wenn der Staat X den Attentäter A auffordert, alle „,missliebigen“ Schriftsteller zu ermorden. Letztlich dürfte es hier auf den Einzelfall ankommen: Ist der Kreis der „missliebigen Schriftsteller" hinreichend präzise, so dass diese individualisierbar sind, dürfte ein Auftrag vorliegen; ist diese Frage zu verneinen, dürfte der Auftrag jedoch nicht hinreichend konkretisiert sein, so dass eine Zurechnung ausscheiden muss.

- Diese Voraussetzungen des Vorliegens einer „Beauftragung“ im Sinne der Zurechnungsregel des Art. 8 ILC-Entwurfs implizieren auch, dass eine Art „Kettenbeauftragung ${ }^{613}$ grundsätzlich möglich ist, so dass jedenfalls in dem Fall, in dem das zu erreichende Ziel und der Beauftragte hinreichend präzisiert bzw. individualisiert sind, auch eine Zurechnung des Verhaltens von „Helfern“ des Auftraggebers möglich ist $^{14}$.

- Da die Beauftragung in Art. 8 ILC-Entwurf neben der zweiten Konstellation der Leitung oder Kontrolle des jeweiligen Verhaltens steht, setzt die erste Alternative des Art. 8 ILC-Entwurf - im Einklang mit der Staatenpraxis ${ }^{15}$ - gerade nicht voraus, dass der Staat die Tätigkeit der agierenden Person(en) auch kontrolliert; vielmehr ist eine Beauftragung im dargelegten Sinn eine zwar notwendige, auf der anderen Seite aber auch eine hinreichende Voraussetzung für die Zurechenbarkeit des jeweiligen Verhaltens.

- Die „Beauftragung“ kann - entsprechend den auch sonst diesbezüglich zum Zuge kommenden völkerrechtlichen Regeln - nicht nur ausdrücklich, sondern auch konkludent erfolgen ${ }^{16}$, wobei diesfalls jeweils nachzuweisen sein wird, dass eine solche konkludente Beauftragung auch tatsächlich erfolgte, was nicht immer einfach sein dürfte.

Vgl. in diese Richtung auch ILC, Kommentar, YbILC 1974 II 1, 283, Ziff. 1; IGH Rep. 1980, 3, Ziff. 57 ff. (Teheraner Geiselfall); 16 Iran-USCTR, 76 ff. (Short). Allerdings kann ein solches Verhalten des Staates X aus anderen Gründen ein völkerrechtliches Delikt darstellen, z.B. wegen eines Verstoßes gegen menschenrechtliche Verpflichtungen.

Vgl. diesen Ausdruck bei Griebel, de facto-Organe (Fn. 1), 169.

In diese Richtung wohl auch RIAA VIII, 84 (Black Tom and Kingsland).

Vgl. so wohl RIAA VIII, 84 (Black Tom and Kingsland); IT-94-1-A, ICTY, Appeals Chamber, Judgement, 15.7.1999 (Tadic).

Ebenso etwa Griebel, de facto-Organe (Fn. 1), 168. 
- Sehr problematisch ist die Frage der Reichweite der Zurechnung im Falle einer Beauftragung, ein Problem, das gelegentlich auch unter dem Stichwort der „,quasi-ultra vires-Handlungen“ erörtert wird ${ }^{17}$. M.E. handelt es sich hier - im Gegensatz zu der von Art. 7 ILC-Entwurf erfassten Situation - aber gerade nicht um eine Frage des ultra vires-Verhaltens, sondern um (wie erwähnt) die Reichweite der Zurechnung des Verhaltens des Beauftragten. Denn die Frage nach der Zurechnung von ultra viresHandlungen stellt sich von Vornherein immer nur dann, wenn eine Person als solche aufgrund ihrer Stellung im Staatsapparat als Staatsorgan anzusehen ist und daher alle durch sie in dieser Funktion gesetzten Handlungen (aber auch Unterlassungen) dem Staat zuzurechnen sind, ohne dass es eines irgendwie gearteten individuellen Auftrags oder einer Kontrolle bedürfte. Geht es hingegen um eine Beauftragung, so werden nicht alle von einer Person in ihrer Eigenschaft als Staatsorgan gesetzten Handlungen dem Staat zugerechnet, sondern eben nur diejenigen, auf die sich der Auftrag bezieht. Insofern kann es bei einer Beauftragung von Vornherein kein ultra vires-Handeln geben.

So dürfte auch der ILC-Entwurf auszulegen sein, aus dem sich bereits aufgrund der systematischen Stellung des Art. 7 ILC-Entwurf nach Art. 4 ILC-Entwurf, jedoch vor Art. 8 ILC-Entwurf, ergibt, dass Art. 7 ILC-Entwurf Art. 4 ILC-Entwurf ergänzen soll ${ }^{18}$. Auch in den Kommentierungen der ILC zu Art. 7, 8 ILC-Entwurf wird die Frage von ultra vires-Handlungen von de facto-Organen nicht angesprochen, was vor dem Hintergrund des Gesagten nur konsequent ist.

Damit ist also bei der Frage nach der Reichweite der Zurechnung des Verhaltens eines Beauftragten letztlich entscheidend, ob die jeweilige Handlung (noch) vom Auftrag erfasst wird, wobei diese Erfassung sowohl auf der ausdrücklichen Erwähnung als auch auf einem konkludenten „Mitdenken“ beruhen kann. M.a.W. geht es also um die Auslegung des erfolgten Auftrags. Hierbei ist - im Anschluss an die auch sonst im Recht der Staatenverantwortlichkeit zum Zuge kommende objektive Sichtweise ${ }^{19}$ darauf abzustellen, ob der beauftragende Staat vernünftigerweise damit rechnen musste, dass die Ausführung des Auftrags das entsprechende Verhalten des Beauftragten nach sich zieht bzw. mit sich bringt, dies im Übrigen unabhängig davon, ob der Auftraggeber dieses Verhalten ausdrücklich oder konkludent angeordnet oder gar verboten hat: Denn der Zurechnungsgrund besteht ja gerade in der Beauftragung, so dass sich der Auftraggeber all diejenigen Verhaltensweisen des Beauftragten zurechnen lassen muss, die mit dem Auftrag vernünftigerweise im Zusammenhang stehen oder stehen können bzw. die anlässlich der Ausführung des Auftrages erfolgen. Insofern spielt hier durchaus auch der Grundgedanke des Art. 7 ILC-Entwurf, wenn auch auf einer anderen Ebene:

Vgl. Griebel, de facto-Organe (Fn. 1), 168 f.

So denn auch Crawford, First Report on State Responsibility, A/CN.4/490/Add.5, Ziff. 166.

Vgl. in Bezug auf die Rolle eines „Verschuldens“ im Recht der Staatenverantwortlichkeit bereits Epiney, Völkerrechtliche Verantwortlichkeit (Fn. 4), 77 ff.; aus neuerer Zeit zu dieser Frage etwa Oliver Diggelmann, Fault in the law of State Responsibility - pragmatism ad infinitum, GYIL 2006, 293 ff.; Felder, Beihilfe (Fn. 3), 78 ff. 
Wenn der Staat sich Privater bedient, obliegt es ihm, die geeigneten Vorkehrungen (etwa bei der Auswahl der Personen oder der Kontrolle der Tätigkeiten) zu treffen, damit diese das Mandat so wie im Auftrag umschrieben ausführen, so dass seine Verantwortlichkeit nicht mit dem Argument des excès de pouvoir verneint werden $\mathrm{kann}^{20}$.

Diese Grundsätze seien wiederum an dem Beispiel der Beauftragung des Attentäters A durch den Staat X, den Schriftsteller S umzubringen, illustriert: Kommt es bei dem Attentat auch zum Tod anderer unbeteiligter Personen, so sind auch diese Handlungen X grundsätzlich zuzurechnen, da im Falle der Beauftragung eines Attentäters ein Risiko des Todes Unbeteiligter zu gegenwärtigen ist. Gleiches dürfte für den Fall gelten, dass A versehentlich nicht S, sondern den Unbeteiligten U umbringt: Auch hier handelt es sich um eine anlässlich des Auftrages begangene Tat bzw. um einen Irrtum, mit der / dem letztlich gerechnet werden muss. Hingegen wäre eine Zurechnung dann zu verneinen, wenn A zur Tötung des S ein Flugzeug entführen und in die Luft sprengen würde, da diese Tat vernünftigerweise nicht mehr im Zusammenhang mit der geplanten Tötung des S steht.

Insgesamt wirft die Konstellation der Beauftragung - abgesehen von dem zuletzt angesprochenen Punkt - relativ wenige Auslegungsprobleme auf, was wohl auch und gerade daraufhin zurückzuführen ist, als es hier wohl um den „Prototyp“ eines de facto-Organs geht, zieht der Staat doch zur Erreichung eines bestimmten Ziels ausdrücklich oder konkludent Private heran, die er mit bestimmten Missionen betraut.

\section{Staatliche Leitung oder Kontrolle}

Bei der zweiten Fallgruppe in Art. 8 ILC-Entwurf wird eine staatliche Leitung oder Kontrolle („direction or control“) des jeweiligen Verhaltens durch den Staat vorausgesetzt. Auch diese Konstellation ist in der Staatenpraxis im Grundsatz anerkannt ${ }^{21}$, wobei jedoch die Präzisierung der genauen Voraussetzungen, bei deren Vorliegen tatsächlich von einer Kontrolle oder Leitung im Sinne dieser Bestimmung gesprochen werden kann, einigen Schwierigkeiten unterliegt.

Insbesondere fragt es sich, wie genau bzw. wie weitgehend die staatliche Kontrolle oder Leitung des in Frage stehenden Verhaltens ausgestaltet sein muss. Da dieser Zurechnungsgrund - im Gegensatz zu Art. 4-6 ILC-Entwurf - keine formelle oder materielle Betrauung oder Beauftragung der Privaten (mit der Ausübung hoheitlicher Gewalt) voraussetzt, kommt lediglich die faktische Kontrolle des privaten Verhaltens als Anknüpfungspunkt in Betracht, so dass Art. 8 2. Alt. ILC-Entwurf ein gewisses Mindestmaß

20 I. Erg. ebenso m.w.N. Griebel, de facto-Organe (Fn. 1), 213 f.; Claus Kress, Gewaltverbot und Selbstverteidigungsrecht nach der Satzung der Vereinten Nationen bei staatlicher Verwicklung in Gewaltakte Privater, 1995, 135; Wolfrum, in: Essays in Memory of Oscar Schachter (Fn. 6), 423 (431 f.). In diesem Sinn ist wohl auch das Urteil in IT-94-1-A, ICTY, Appeals Chamber, Judgement, 15.7.1999 (Tadic), Ziff. 119, auszulegen, wo die Kammer davon spricht, dass auch eine Überschreitung des Auftrages zurechenbar sei, wenn sich diese anlässlich der Ausführung des Auftrags ergibt.

21 S. insbesondere ICJ Rep. 1980, 3, Ziff. 58 ff. (Teheraner Geiselfall); ICJ Rep. 1986, 14, Ziff. 103 ff. (Military and Paramilitary Activities in and against Nicaragua). 
an effektiver Kontrolle über das Geschehen durch den Staat voraussetzt. So reichen insbesondere eine allgemeine Abhängigkeit der jeweiligen Privaten von dem betreffenden Staat oder die allgemein gehaltene staatliche Unterstützung nach der einschlägigen internationalen Praxis für eine Zurechnung des Privatverhaltens nicht aus. Erforderlich ist zumindest eine tatsächliche Kontrolle über das jeweilige Agieren der Privaten, ohne dass sich diese Kontrolle auf jedes einzelne Element des privaten Handelns beziehen muss.

Im viel zitierten Nicaragua-Fall verlangte der IGH den Beweis, dass die USA eine "effective control of the military or paramilitary operations in the course of which the alleged violations were committed" 22 hatten. Dieser strenge Maßstab wurde von der Berufungskammer des ICTY später etwas gelockert. In ihrem Urteil zum TadicFall liess sie eine "overall control going beyond the mere financing and equipping of such forces and involving also participation in the planning and supervision of military operations ${ }^{, 23}$ genügen, wobei Vieles dafür spricht, dass sich diese Aussagen auf den spezifischen Bereich des humanitären Völkerrechts beschränken ${ }^{24}$.

Im Ergebnis und im Anschluss an die Ausführungen im Zusammenhang mit der Beauftragung $^{25}$ können die Anforderungen an das Ausmaß der Kontrolle wie folgt umschrieben werden:

- Jedenfalls nicht ausreichend sind Unterstützungshandlungen, selbst wenn diese sehr umfangreich ausfallen und die Durchführung der in Frage stehenden Handlungen gar erst ermöglichen ${ }^{26}$. A fortiori muss dies für Handlungen gelten, die lediglich im Interesse eines Staates liegen (könnten).

- $\quad$ Auf der anderen Seite muss der jeweilige Staat nicht zwingend jede einzelne Handlung des de facto-Organs kontrollieren; vielmehr reicht eine grundsätzliche Kontrolle über deren Aktivitäten. Denn abgesehen davon, dass es kaum möglich sein dürfte, dass der Staat auch über jede einzelne Handlung tatsächlich die Herrschaft innehat, muss es nach der ratio der Zurechnung auf der Grundlage des Art. 8 ILC-Entwurf ausreichen, dass über die grundsätzliche Ausrichtung des jeweiligen Verhaltens eine Kontrolle gegeben ist. Dass das de facto-Organ in diesem Rahmen dann auch „autonom“ agieren kann, ohne dass die Zurechnungskette unterbrochen wird, erscheint - letztlich parallel wie im Rahmen der Beauftragung - schon insofern zwingend, als es im Verantwortungsbereich des Staates liegen muss, auch diejenigen Handlungen zu verantworten, die im Rahmen der grundsätzlichen Steuerung der Tätigkeit gesetzt werden.

- $\quad$ Allerdings muss - in Anknüpfung auch an die Unterscheidung zur Beauftragung und vor dem Hintergrund der Abgrenzung zu bloßen Unterstützungshandlungen - die Kontrolle auf der anderen Seite insofern effektiv sein, als der Staat das entsprechende Verhalten grundsätzlich ,im Griff“ hat, so dass er letztlich die Operation steuern kann, was insbesondere implizieren dürfte, dass er über die Ausrichtung des jeweiligen

ICJ Reports 1986, 14, Ziff. 64 (Nicaragua).

ICTY, Appeals Chamber Judgement, Case IT-94-1-A, 15.7.1999, Ziff. 145 (Tadic).

Vgl. ebenso Griebel, De facto-Organe (Fn. 1), 170 f.

S.o. II.1.

Allerdings kann das Verhalten des unterstützenden Staates aus anderen Gründen ein völkerrechtliches Delikt darstellen. Vgl. in diesem Zusammenhang zur Beihilfe zum Völkerrechtsdelikt Felder, Beihilfe (Fn. 3), 2007, passim. 
Verhaltens bestimmt und dieses auch grundsätzlich beenden kann. Ist diese Schwelle nicht erreicht, so besteht kein Grund, die für das Bestehen der Zurechnung doch hohe Schwelle der „Steuerung“ des privaten Verhaltens anzunehmen, geht es bei der Zurechnung doch gerade darum, dass die jeweilige Person in Bezug auf das betreffende

Verhalten den Staat „repräsentiert“.

Damit dürfte die hier vertretene Sicht auf der einen Seite nicht die Kontrolle aller Einzelakte verlangen; auf der anderen Seite dürfte eine eher diffuse overall control ebenfalls nicht ausreichen $^{27}$, da hiermit die Notwendigkeit einer effektiven Kontrolle zu sehr relativiert wird; diese ist aber zur Begründung einer hinreichend engen Verbindung zum Staat notwendig. Die Abgrenzung kann hier aber Schwierigkeiten bereiten; immerhin könnte eine Leitlinie die Frage nach der Steuerungsfähigkeit des Staates sein: Nur wenn dieser in der Lage ist, das in Frage stehende Verhalten auch tatsächlich zu beeinflussen und es auch tatsächlich grundsätzlich steuert, was auch bedingt, dass er es beenden können muss, sind die Voraussetzungen einer effektiven Kontrolle im Sinne des Art. 8 ILC-Entwurf gegeben.

Weiter kommt eine Zurechnung auf der Grundlage des Art. 8 ILC-Entwurf von Vornherein nur unter der Voraussetzung in Betracht, dass ein zeitlich der Handlung vorgelagerter Kontakt zum Staatsapparat gegeben ist ${ }^{28}$ : Denn nur unter dieser Voraussetzung kann eine Kontrolle tatsächlich stattfinden; im Falle nachträglicher „Zustimmung“ oder „Übernahme“ des in Frage stehenden Verhaltens geht es gerade nicht um eine Kontrolle der Aktion, sondern um andere, noch zu erörternde ${ }^{29}$ Zurechnungsgründe.

Schließlich ist darauf hinzuweisen, dass - unter der Voraussetzung der effektiven Kontrolle im dargelegten Sinn - auch ein Unterlassen zugerechnet werden kann ${ }^{30}$.

\section{Abgrenzung zu anderen Zurechnungsgründen bzw. Formen der völkerrechtlichen Verantwortlichkeit}

Die Rechtsfigur des de facto-Organs im Sinne des Art. 8 ILC-Entwurf ist von anderen Formen der Zurechnung ,eigentlich“ nicht staatlichen Verhaltens bzw. von der Verantwortlichkeit im Zusammenhang mit dem Verhalten Privater abzugrenzen, wobei insbesondere drei Konstellationen von Bedeutung sind: Verhalten bei Abwesenheit oder Ausfall staatlicher

27 Vgl. i. Erg. ähnlich wohl Griebel, de facto-Organ (Fn. 1), 172 f.; m.w.N. wohl auch Ziegler, Fluchtverursachung (Fn. 2), 125 ff., wenn diese auch stärker dazu tendiert, eine overall control ausreichen lassen zu wollen. Vgl. auch den Kommentar der ILC zu Art. 8, die zu Recht auf die Notwendigkeit einer Prüfung im Einzelfall abstellt und ausführlich auf die diesbezügliche völkerrechtliche Judikatur hinweist, vgl. ILC, Commentary, UN Doc. A/56/10, IV.E.2, Ziff. 5 zu Art. 8 mit Fußnote 169. 123.

29 S. sogleich unten III.

30 Ziegler, Fluchtverursachung (Fn. 2), 124. 
Stellen (1.), die Übernahme privaten Verhaltens (2.) und die Verantwortlichkeit der Staaten im Zusammenhang mit dem Verhalten Privater (3. $)^{31}$.

\section{Verhalten bei Abwesenheit oder Ausfall staatlicher Stellen}

Art. 9 ILC-Entwurf sieht vor, dass im Falle einer Abwesenheit oder des Ausfalls der staatlichen Stellen (,,failed state") das Verhalten von Personen oder Personengruppen ebenfalls einem Staat zurechenbar ist, wenn diese Personen faktisch hoheitliche Befugnisse ausüben und besondere Umstände die Ausübung dieser Befugnisse notwendig machen, womit die Parallelität dieser Fallkonstellation mit der Geschäftsführung ohne Auftrag im nationalen Recht deutlich wird. Obwohl keine ,rechtliche Ermächtigung“ existiert, rechtfertigen besondere Umstände die Ausübung öffentlicher Funktionen im Interesse des Staates. Auch dieser Zurechnungsgrund ist in der völkerrechtlichen Praxis anerkannt, wenn die diesbezüglichen Fälle auch relativ selten sind ${ }^{32}$.

Im Gegensatz zu der von Art. 8 ILC-Entwurf erfassten Konstellation geht es hier also um eine besondere Konstellation, in der letztlich - aufgrund der Abwesenheit staatlicher Gewalt keine (faktische) Einbindung in den Staatsapparat vorliegt, jedoch aufgrund anderer, besonderer Umstände eine Zurechnung anerkannt wird.

\section{2. „ „Übernahme“ privaten Verhaltens}

Kann ein Verhalten einem Staat nicht nach den eben erläuterten Vorschriften zugerechnet werden, anerkennt der Staat dieses jedoch als sein eigenes, so ist die Tätigkeit gemäß Art. 11 ILC-Entwurf im Umfang dieser Übernahme dem Staat zuzurechnen.

\section{Art. 11 ILC-Entwurf}

„Verhalten, das ein Staat als sein eigenes anerkannt und annimmt

Ein Verhalten, das einem Staat nach den vorstehenden Artikeln nicht zugerechnet werden kann, ist gleichwohl als Handlung des Staates im Sinne des Völkerrechts zu werten, wenn und soweit der Staat dieses Verhalten als sein eigenes anerkennt und annimmt ${ }^{33}$.“

Dieser Zurechnungsgrund - der strukturelle Ähnlichkeit mit Art. 8 ILC-Entwurf aufweist - ist in der Staatenpraxis in Bezug auf Handlungen, die einen gewissen „Dauercharakter“

31 Nicht näher erörtert werden sollen im Folgenden die sog. de facto-officials, da diese im Ergebnis unter Art. 4, 5 ILC-Entwurf fallen, vgl. zum Problem Griebel, de facto-Organe (Fn. 1), 166 f.

32 Vgl. ausführlich zu Art. 9 m.w.N. Robin Geiß, „Failed States“. Die normative Erfassung gescheiterter Staaten, 2005, 261 ff.; umfassend zum Problemkreis auch Hinrich Schröder, Die völkerrechtliche Verantwortlichkeit im Zusammenhang mit failed und failing States, 2007, passim.

33 "Article 11. Conduct acknowledged and adopted by a State as ist own. Conduct which is not attributable to a State under the preceding articles shall nevertheless be considered an act of that State under international law if and to the extent that the State acknowledges and adopts the conduct in question as its own." 
aufweisen, anerkannt ${ }^{34}$. Voraussetzung für die Einschlägigkeit dieses Zurechnungsgrundes ist aber jedenfalls, dass der Staat erkennen lässt, dass er mit dem Verhalten der agierenden Personen oder zumindest mit den daraus resultierenden Ergebnissen in jeder Beziehung einverstanden ist und damit das private Verhalten als sein eigenes Verhalten ansieht. Eine bloße Passivität seitens des Staates - wie sie typischerweise im Falle der Verletzung der völkerrechtlichen Pflichten im Zusammenhang mit Aktionen Privater zu beobachten ist ${ }^{35}-$ reicht für eine Zurechnung nicht aus, sondern notwendig ist vielmehr eine entsprechende ausdrückliche oder implizite Stellungnahme des Staates, durch die eine Art Solidarisierung des Staates mit den Handlungen der Privaten zum Ausdruck kommt.

Beispielhaft erwähnt sei aus der Praxis der Teheraner Geiselfall ${ }^{36}$ : Hier kam der IGH zum Schluss, dass das Verhalten der Studenten, die die amerikanische Botschaft überfallen und das Personal als Geiseln festgehalten hatten, dem Staat Iran ab dem Zeitpunkt einer entsprechenden Deklaration des Revolutionsführers Khomeini, dass Iran das Agieren der Studenten in jeder Hinsicht unterstütze, zugerechnet werden könne.

Die Tragweite dieses Zurechnungstatbestandes dürfte jedoch insofern beschränkt sein, als die Staatenpraxis - insbesondere der erwähnte Teheraner Geiselfall - nicht den Schluss erlaubt, dass ein zeitlich abgeschlossenes Verhalten, das privaten Charakter aufweist, durch eine Art staatliche Zustimmung ex posterior als staatliches Verhalten zu qualifizieren wäre. Vielmehr erfasst Art. 11 ILC-Entwurf lediglich das „Umschlagen“ eines zunächst privaten Verhaltens in ein staatliches Verhalten, ohne dass jedoch dem zu Beginn privaten Verhalten ebenfalls staatlicher Charakter zukäme und dieses „umzuqualifizieren“ wäre ${ }^{37}$. Eine Rückwirkung staatlicher Anerkennung bzw. Übernahme auf die Zurechnung bereits abgeschlossener Handlungen kommt daher nicht in Betracht, so dass sich die einmal - zum Zeitpunkt des Verhaltens - getroffene (Nicht-) Zurechnung eines Verhaltens zum Staat nicht aufgrund nach dem betreffenden Verhalten erfolgender staatlicher Akte modifizieren lässt.

Damit wird auch das Verhältnis zu Art. 8 ILC-Entwurf deutlich: Während es bei Art. 8 ILCEntwurf darum geht, dass ein an sich privates Verhalten aufgrund einer Beauftragung oder der Kontrolle durch den Staat als staatliches Verhalten angesehen wird, wobei eine gewisse faktische Einbindung der agierenden Personen in den Staatsapparat von Bedeutung ist, ist der Hintergrund der Zurechnung bei Art. 11 ILC-Entwurf in der Identifizierung oder eben Übernahme an sich in jeder Beziehung privaten Verhaltens durch den Staat anzusehen, so dass Art. 11 ILC-Entwurf zu einer Umqualifizierung privaten Verhaltens führt. Gerade dieser letzte Aspekt ist wohl auch entscheidend dafür, dass dieser Zurechnungsgrund nur bei

\footnotetext{
34 Vgl. ausführlich bereits Epiney, Völkerrechtliche Verantwortlichkeit (Fn. 4), 162 ff.

35 S. hierzu unten III.3.

36 ICJ, United States Diplomatic and Consular Staff in Teheran, ICJ Rep. 1980, 1 ff.

37 Vgl. ausführlich schon Epiney, Völkerrechtliche Verantwortlichkeit (Fn. 4), 179 ff.; ebenso Griebel, de facto-Organe (Fn. 1), 83 ff., 173 ff., 222 ff. (mit Hinweisen auf abweichende Ansichten), sowie Ziegler, Fluchtverursachung (Fn. 2), 131 ff., die aber den besonderen Zurechnungsgrund der Übernahme privaten Verhaltens für überflüssig hält und die entsprechenden Fallgestaltungen über Art. 8 ILC-Entwurf (de facto-Organ) lösen will, wobei sie einen parallelen Maßstab wie im Rahmen des Art. 8 ILC-Entwurf anlegen will. A.A. wohl Tal Becker, Terrorism and the State. Rethinking the Rules of State Responsibility, 2006, 72 ff.; unklar Wolfrum, in: Essays in Memory of Oscar Schachter (Fn. 6), 423 (426).
} 
Verhalten mit Dauercharakter, nicht hingegen bei abgeschlossenen Verhaltensweisen, zum Zuge kommen kann.

Verdeutlicht werden kann dies etwa am Beispiel der Zurechnung des Verhaltens privater Sicherheitsfirmen: Auch hier kann Art. 11 ILC-Entwurf nur für Verhaltensweisen mit einem gewissen Dauercharakter, wie z.B. Entführungen oder Gefangenenhaltung, in Betracht kommen. Hingegen kann Art. 11 ILC-Entwurf nicht in Bezug auf bereits abgeschlossenes Verhalten greifen, so dass etwa die staatliche Beauftragung eines privaten Sicherheitsunternehmen, dessen Angestellte vor dieser Beauftragung bei der Erfüllung anderer Aufträge schwere Straftaten begangen haben, nicht zur Zurechnung dieses zeitlich vorgelagerten Verhaltens führen kann ${ }^{38}$.

Insgesamt handelt es sich damit bei Art. 11 ILC-Entwurf insofern um einen nicht nur im Verhältnis zu Art. 8 ILC-Entwurf, sondern auch im Verhältnis $\mathrm{zu}$ den anderen Zurechnungsgründen speziellen Zurechnungstatbestand, als der Hintergrund und die Begründung der Zurechnung nicht in einer irgendwie gearteten „Herrschaft“ des Verhaltens der agierenden Personen durch den Staat - sei es durch die de iure oder de facto Einbindung in den Staatsapparat oder die Kontrolle der privaten Tätigkeiten, sei es durch eine irgendwie geartete Beauftragung - geht, sondern die Zurechnung wird allein durch die „Anerkennung“ einer bereits begonnenen und weiter andauernden Handlung als eigenes Verhalten begründet $^{39}$. Dies erscheint insofern auch begründet, als es den Staaten freistehen sollte, (zunächst) privates Verhalten als eigenes zu übernehmen. Dieser Hintergrund der Übernahme privaten Verhaltens impliziert aber auf der anderen Seite auch, dass - wie auch in der Formulierung des Art. 11 ILC-Entwurf zum Ausdruck kommt - eine Übernahme nur insoweit in Betracht kommt, als der Staat diese tatsächlich (ausdrücklich oder konkludent) zum Ausdruck bringt, so dass ein bloßes Ausnutzen einer durch Private geschaffenen Situation (wie etwa im Anschluss an Entführungen) nicht ausreicht und eine Zurechnung weiterer Verhaltensweisen der betreffenden Personen, die nicht von der Übernahme erfasst sind, auf der Grundlage des Art. 11 ILC-Entwurf nicht in Betracht kommt ${ }^{40}$. Dies ändert jedoch nichts daran, dass eine Zurechnung aufgrund des Art. 8 ILC-Entwurf gegeben sein kann, falls dessen Voraussetzungen vorliegen.

Falls also ein Staat bei einer Entführung zum Ausdruck bringt, dass er das weitere Festhalten der Personen in jeder Beziehung als sein eigenes Verhalten ansieht, führt dies zur Zurechnung des Verhaltens der Entführer, nur soweit das Festhalten der jeweiligen Personen betroffen ist. Eine Tötung der Entführten durch die Entführer kann hingegen nur dann dem betreffenden Staat zugerechnet werden, wenn er das Verhalten der Entführer effektiv im Sinne des Art. 8 ILC-Entwurf kontrolliert, es sei denn, der Staat bringe in der „Übernahmeerklärung“ zum Ausdruck, dass er alle Akte der Entführer, also einschliesslich einer Tötung der Entführten, als seine eigenen ansehen wolle.

\section{Zu staatlichen Pflichten im Zusammenhang mit dem Verhalten Privater}

\footnotetext{
38 Allerdings können bei einer solchen Konstellation andere Pflichten verletzt worden sein, hierzu unten III.3.

39 Eine eigentliche Kontrolle des privaten Verhaltens ist damit nicht vorausgesetzt, unklar in dieser Beziehung noch Epiney, Völkerrechtliche Verantwortlichkeit (Fn. 4), 189.

40 A.A. insoweit Griebel, de facto-Organe (Fn. 1), $174 \mathrm{f}$.
} 
Führt eine Analyse der Voraussetzungen der im Zusammenhang mit einem bestimmten Verhalten in Betracht kommenden Zurechnungsgründe $\mathrm{zu}$ dem Ergebnis, dass kein Zurechnungsgrund zu greifen vermag, so dass das in Frage stehende Verhalten nicht als staatliches Verhalten angesehen werden kann, kann sich eine völkerrechtliche Verantwortlichkeit der Staaten gleichwohl wegen der Verletzung eigener Verhaltenspflichten im Zusammenhang mit dem Verhalten Privater ergeben. Voraussetzung dafür ist jedoch, dass dem Staat in Bezug auf das Verhalten der privaten Sicherheitsunternehmen eine eigene völkerrechtliche Primärpflicht obliegt, deren Verletzung zu bejahen ist ${ }^{41}$. Von besonderer Bedeutung ist hier die in verschiedenen Bereichen bestehende völkerrechtliche Pflicht der Staaten, die nach den Umständen erforderliche Sorgfalt anzulegen (due diligence), damit Private von ihrem Territorium aus nicht bestimmte Rechtsgüter beeinträchtigen (z.B. die territoriale Integrität anderer Staaten oder Menschenrechte). Vorausgesetzt wird hier immer die Existenz einer entsprechenden primärrechtlichen Pflicht, so dass es hier - im Gegensatz zu Art. 8 ILC-Entwurf - in erster Linie um die Reichweite der entsprechenden primärrechtlichen Pflicht geht.

Die Tragweite solcher „due-diligence-Pflichten“ der Staaten ${ }^{42}$ ist in der Regel insoweit eingeschränkt, als es sich um nicht um Ergebnis-, sondern um Handlungspflichten handelt und von den Staaten nur faktisch mögliches Verhalten verlangt werden kann (wobei allerdings eine Mindestorganisation der Staaten vorausgesetzt wird), so dass es zu berücksichtigen ist, wenn ein Staat - z.B. aufgrund innerer Unruhen - nicht in der Lage ist, einen objektiv grundsätzlich geforderten Standard einzuhalten. Grundsätzlich müssen die Staaten solchen Präventionspflichten aber mit der gebotenen Sorgfalt nachkommen, wobei das Maß der gebotenen Sorgfalt nach den Umständen variieren kann. Die ergriffenen Maßnahmen müssen in jedem Fall grundsätzlich geeignet sein, die entsprechenden privaten Verhaltensweisen zu verhindern bzw. es erlauben, auf tatsächlich erfolgte Verletzungen effektiv zu reagieren.

\section{Schluss: zur Rolle der Rechtsfigur des de facto-Organs im Recht der Staatenverantwortlichkeit}

Die Zurechnungsregeln für de facto-Organe im Sinne des Art. 8 ILC-Entwurf und für die Übernahme staatlichen Verhaltens im Sinne des Art. 11 ILC-Entwurf können auf der Grundlage der erfolgten Ausführungen wie folgt systematisiert werden:

- $\quad$ Findet das betreffende staatliche Verhalten, das die Zurechnung begründen könnte, vor dem (möglicherweise) zuzurechnenden Verhalten statt, so kommt der Maßstab der Beauftragung (Art. 8 ILC-Entwurf, 1. Alternative) zum Zuge.

- $\quad$ Die Voraussetzung der effektiven Kontrolle des in Frage stehenden Verhaltens (Art. 8 ILC-Entwurf, 2. Alternative) bezieht sich auf staatliche Verhaltensweisen, die letztlich

41 Ausführlich hierzu Epiney, Völkerrechtliche Verantwortlichkeit (Fn. 4), 205 ff.; Wolf, Haftung der Staaten (Fn. 4), 234 ff.; Ziegler, Fluchtverursachung (Fn. 2), 156 ff.; s. auch Felder, Beihilfe (Fn. 3 ), 73 ff.

42 Vgl. ausführlich zu diesen Pflichten und zur Präzisierung des due diligence-Massstabes Epiney, Völkerrechtliche Verantwortlichkeit (Fn. 4), 205 ff. 
während der jeweiligen (möglicherweise) zuzurechnenden Handlungen gesetzt werden müssen.

- $\quad$ Und die Übernahme staatlichen Verhaltens kommt durch „,nachträgliches“ staatliches Verhalten in engen Grenzen in Bezug auf Dauerdelikte zum Zuge.

Speziell in Bezug auf die Zurechnung auf der Grundlage von Art. 8 ILC-Entwurf zeigen die angestellten Überlegungen zwar die teilweise bestehenden Schwierigkeiten bei der Auslegung und Anwendung dieses Zurechnungstatbestandes ${ }^{43}$; hinzukommen in der Praxis regelmäßig auftretende Beweisprobleme. Gleichzeitig zeigt jedoch eine nähere Analyse der Voraussetzungen des Art. 8 ILC-Entwurf, dass diese durchaus präzisiert werden können, woran auch ihre Anwendungsbedürftigkeit auf den Einzelfall und damit die in gewissem Sinn bestehende Maßgeblichkeit (auch) der Umstände des Einzelfalls nichts zu ändern vermag. Insofern verdient dieser Zurechnungsgrund durchaus verstärkte Beachtung, auch und gerade im Zusammenhang mit manchen sich stellenden neueren Phänomenen.

Aufgezeigt werden kann dies etwa am Beispiel privater Sicherheitsfirmen: Hier dürfte Art. 8 ILC-Entwurf immer dann einschlägig sein, wenn sich ein Befehlshaber während eines bewaffneten Konfliktes eines privaten Unternehmens bedient, um eine ,heikle“ Operation durchzuführen, oder wenn private Sicherheitsunternehmen faktisch in die Aufrechterhaltung der öffentlichen Ordnung eingebunden werden. Weiter sind alle Fälle „versteckter Aufträge“ seitens des Staates an private Sicherheitsunternehmen unter Art. 8 ILC-Entwurf zu subsumieren.

Insgesamt wäre daher zu wünschen, dass die Staaten diesen Zurechnungsgrund verstärkt einbeziehen, sowohl was ihr eigenes Verhalten als auch was die Geltendmachung von Völkerrechtsdelikten durch andere Staaten betrifft. Denn der in der Staatenpraxis schon lange anerkannte Grundgedanke der Rechtsfigur des de facto-Organs, dass sich ein Staat nicht durch den Rückgriff auf „Private“ seiner völkerrechtlichen Verantwortung entziehen können soll, ist nach wie vor aktuell und verdient Beachtung, die dann ihrerseits wiederum zu einer Präzisierung der Voraussetzungen einer Zurechnung auf der Grundlage des Art. 8 ILCEntwurf beizutragen vermag.

43 Zumal die in Art. 8 ILC-Entwurf im Einklang mit dem geltenden Völkerrecht formulierten Voraussetzungen der Zurechnung recht streng formuliert sind. Vgl. für eine Kritik und für Vorschläge einer Ausweitung der Zurechnung des Verhaltens von de facto-Organen de lege ferenda Griebel, de facto-Organe (Fn. 1), 179 ff. 